\title{
Mathematical models for researching the human factors on the heat transfer in a pool
}

\author{
Jidong $\mathrm{Li}^{1, \mathrm{a}}$ \\ ${ }^{1}$ North China Electric Power University, Baoding 071000, China; \\ a759636597@qq.com
}

Keywords: RNG $k-\varepsilon$ Model, Temperature Field, Equivalent Size, Numerical Simulation.

\begin{abstract}
In order to obtain the temperature field of water in a pool with a person inside, this paper established mathematical models by taking the human factors, including static and dynamic factors, into consideration. Firstly, based on the three-dimensional N-S equations and the concept of the equivalent size, the shape of person is simplified and the model is built by changing some boundary conditions to gain the temperature distribution of water in the pool. Furthermore, the optimal model, which takes the motions of human into account, is developed. The RNG $k-\varepsilon$ model is introduced and improved to accord with our problem. In addition, the result is carried out by means of numeral simulation.
\end{abstract}

\section{Introduction}

Some previous papers and magazines mainly focus on developing the mathematical model based on the finite element analysis to solve the heat transfer problem. In addition, three dimensional differential equations are applied to simulate the heat process and optimize the control of heating container[1]. J. X. Zhang did some researches on the influence for swimming pool water temperature field in different heating modes. One of the modes is traditional heating mode, the other is called floor heating mode. Results illustrate that the swimming pool's temperature field with floor heating mode is more homogeneous than the traditional heating mode, time to achieve the stable temperature field is shorter[2].

\section{Analysis of The Problem}

Human factors include the shape/volume/temperature of the person in the pool, and the motions made by the person.

\section{Static Factors}

Firstly, we research the influence generated from the person's shape, volume and temperature which is call static factors here. The model of the human body[3] is simplified as a cuboid whose value of its length, width and height could reflect the person's volume and the proportion of its length, width and height determines the person's shape. As for the temperature of the person, we regard it as a fixed boundary condition due to the characteristic of the human body's constant temperature.

The static posture of the person in the pool could be simplified as below:

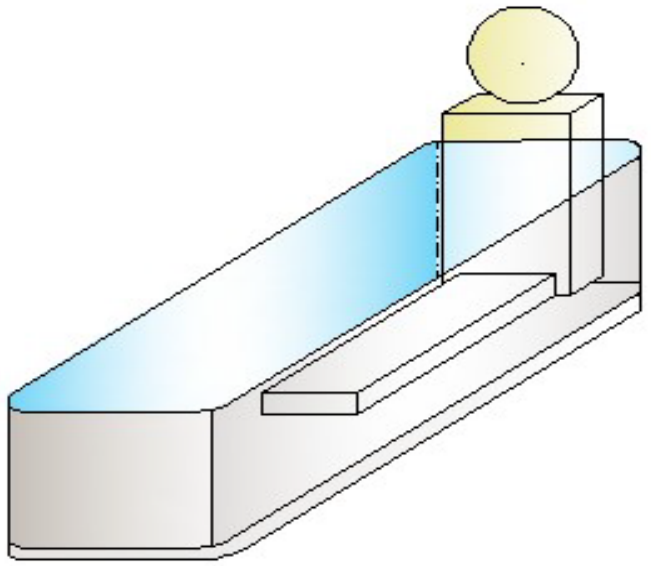

Fig 1 the static posture of the person in the pool 
Different from the simulation of the basic model, in this case, the volume the person occupies should be deprived from the volume of the water before simulating. Based on this irregular volume, we simulate the temperature field.

According to the concept of the equivalent size in fluid mechanics, the irregular volume can be transformed into a cuboid. Meanwhile, two surfaces in constant temperature on the back and bottom sides of the cuboid are introduced. In terms of the transformed volume, we simulate the temperature field as well.

The two temperature fields are presented as follows:
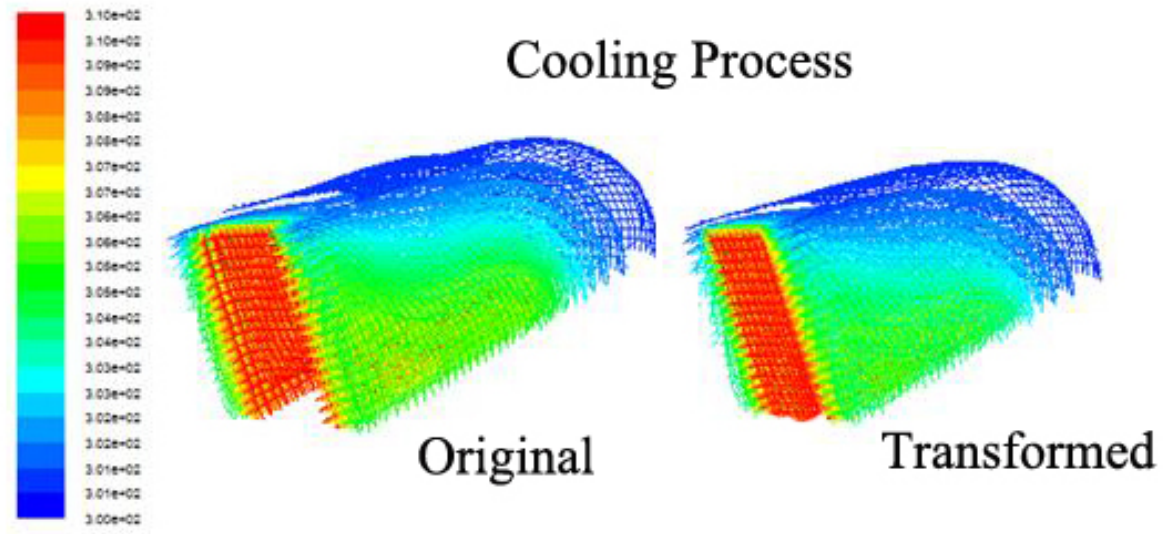

Fig 2 the temperature field with the person inside in cooling process
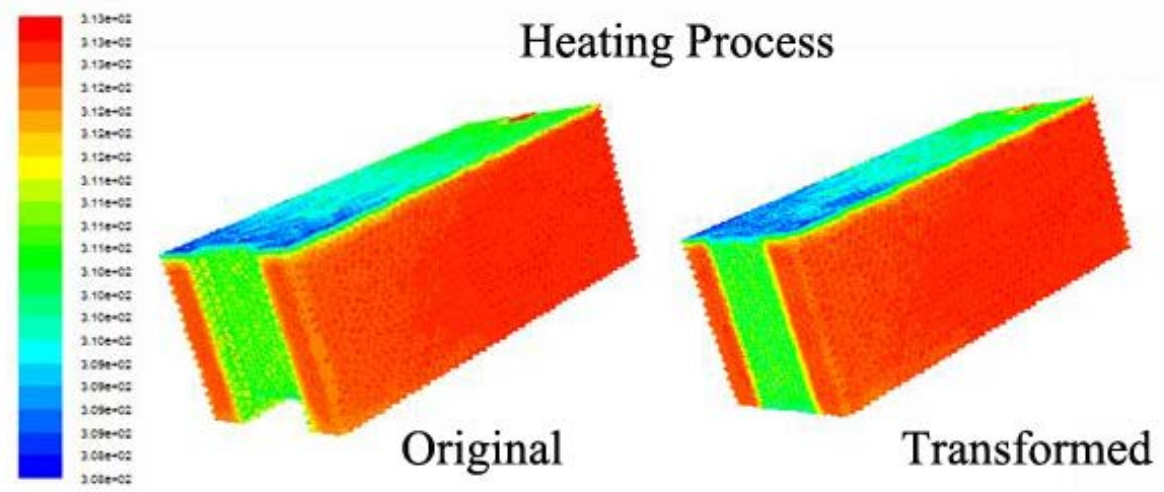

Fig 3 the temperature field with the person inside in heating process

From the figure we can see that the temperature distributions of the irregular volume and the transformed volume are similar to a large extent in both cooling and heating process.

Through the contrast of the temperature fields with and without person in pool, it is further evidence that the method of transforming volumes into regular cuboid based on the equivalent size is effective and accurate whether in cooling or heating process.

\section{Dynamic Factors}

In this section, the motions of the person are taken into account. Considering that the person's motions are irregular and uncertain but limited, the model is simplified by regarding the motions as a vortex spinning with a constant speed.

Firstly, taking the person's center of gravity as the turning center, a relatively rectangular coordinate is built as below: 


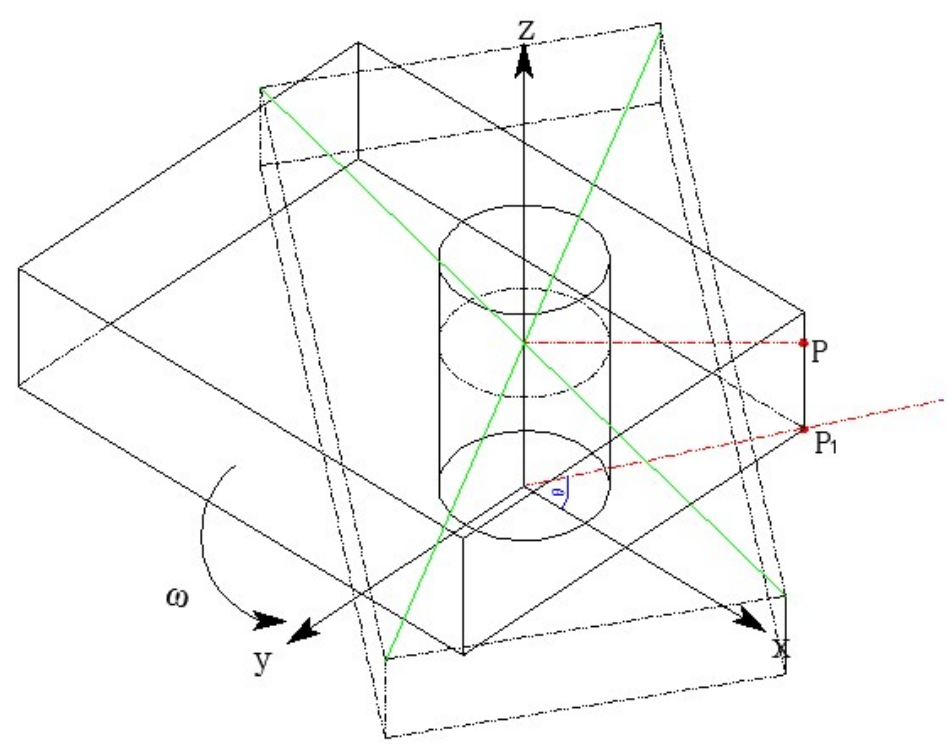

Fig 4 the relatively rectangular coordinate

It is assumed that the three-dimensional flow inside the pool is unsteady and incompressible. In a relative rectangular coordinate, the momentum equation and the continuity equation are listed as follows:

$$
\frac{\partial \bar{v}}{\partial y}+\frac{\partial \bar{u}}{\partial x}=0
$$

The continuity equation is as below:

$$
\frac{\partial(\rho \bar{u})}{\partial \tau}+\frac{\partial(\rho \overline{v u})}{\partial y}=\frac{\partial\left[\mu_{e}\left(\frac{\partial \bar{u}}{\partial y}+\frac{\partial \bar{v}}{\partial x}\right)\right]}{\partial y}-\frac{\partial p}{\partial x}
$$

Next, we introduce the RNG k-cmodel [4] to deal with the turbulence problem. In this model, the turbulent kinetic energy is defined as $k$, the turbulent dissipation rate is defined as $\varepsilon$. The constraint formulas of $k$ and $\varepsilon$ are listed as follows:

$$
\left\{\begin{array}{l}
\frac{\partial(\rho \kappa)}{\partial \tau}+\frac{\partial(\rho \kappa \bar{v})}{\partial y}=\frac{\partial}{\partial y}\left(\alpha_{k} \mu_{e} \frac{\partial \kappa}{\partial y}\right)+\rho\left(P_{k}-\varepsilon\right) \\
\frac{\partial(\rho \varepsilon)}{\partial \tau}+\frac{\partial(\rho \varepsilon \bar{v})}{\partial y}=\frac{\partial}{\partial y}\left(\alpha_{\varepsilon} \mu_{e} \frac{\partial \varepsilon}{\partial y}\right)+\rho \frac{\varepsilon}{\kappa}\left(C_{1 \varepsilon}{ }^{*} P_{k}-C_{2 \varepsilon} \varepsilon\right)
\end{array}\right.
$$

The following equations are supplements of the formula 5-3 for further explanation:

$$
\left\{\begin{array}{l}
C_{1 \varepsilon}{ }^{*}=C_{1 \varepsilon}-\frac{\eta\left(1-\eta / \eta_{0}\right)}{1+\beta \eta^{3}} \\
\eta=(2 E \cdot E)^{1 / 2} \frac{\kappa}{\varepsilon} \\
E=\frac{1}{2}\left(\frac{\partial \bar{u}}{\partial y}+\frac{\partial \bar{v}}{\partial x}\right)
\end{array}\right.
$$

In the model, the values of some constants are listed as follows: $C_{\mu}=0.0845, \alpha_{k}=\alpha_{\varepsilon}=1.39$, $C_{1 \varepsilon}=1.42, C_{2 \varepsilon}=1.42, \eta_{0}=4.377, \beta=0.012$.

We can obtain the velocity fields by solving the RNG k- $\varepsilon$ model. Then the temperature field can be obtained based on the formula 4-2 and the result of the RNG k- $\varepsilon$ model. We figure out the equations by means of the PDE tool in Matlab and obtain the change curve of temperature in time and space. 


\section{Summary}

In terms of the person's shape and volume, the model is simplified by means of regarding the pool with people inside as a whole based on the equivalent .In terms of the person's temperature, considering that the temperature difference between people and water is little and the people's temperature is always constant, the influence on the whole temperature field is neglected. As for the person's motions, the established model is unavailable due to the turbulence motions generate so that the RNG k- $\varepsilon$ model is introduced and improved to solve the problem.

\section{References}

[1] Yuan Wenqing, Chen Xiao, Hu Mingjuan, "Computer Simulation of Three Dimensional Unsteady Temperature Fields during Heating-up Process”, Transactions of Metal Heat Treatment, vol.12,No.2,pp.35-42,June 1991.

[2] Zhang Jinxia, 2014, “The Simulation Analysis of the Influence for Swimming Pool Water Temperature Field in Different Heating Modes” ,PhD diss., Yanshan University., 2014.

[3] Hu Zhi-gang, Cui Hong-li, Guan Xiao-rong, "Simulation research on fluid field of limited space around the human body”, Journal of Machine Design, vol.28,No.5,pp.42-45,May 2011.

[4] ZHANG Shujia, LI Xianhua, ZHU Baolin, ” Applicability of k- $\varepsilon$ Eddy Viscosity Turbulence Models on Numerical Simulation of Centrifugal Pump ”, Journal of Mechanical Enginering,vol.45,No.4,pp.238-242,April 2009. 